Revue Française de Civilisation Britannique

\title{
Introduction : Regards changeants sur l'histoire de la BBC
}

Introduction: Changing Perspectives on the History of the BBC

Lucie de Carvalho, John Mullen et Frédéric Armao

\section{CpenEdition}

\section{Journals}

Édition électronique

URL : https://journals.openedition.org/rfcb/7612

DOI : $10.4000 /$ rfcb.7612

ISSN : 2429-4373

Éditeur

CRECIB - Centre de recherche et d'études en civilisation britannique

Référence électronique

Lucie de Carvalho, John Mullen et Frédéric Armao, « Introduction : Regards changeants sur l'histoire de la BBC $»$, Revue Française de Civilisation Britannique [En ligne], XXVI-1 | 2021, mis en ligne le 02 décembre 2020, consulté le 05 janvier 2022. URL : http://journals.openedition.org/rfcb/7612 ; DOI https://doi.org/10.4000/rfcb.7612

Ce document a été généré automatiquement le 5 janvier 2022.

\section{(i)

Revue française de civilisation britannique est mis à disposition selon les termes de la licence Creative Commons Attribution - Pas d'Utilisation Commerciale - Pas de Modification 4.0 International. 


\title{
Introduction : Regards changeants sur l'histoire de la BBC
}

\author{
Introduction: Changing Perspectives on the History of the $B B C$
}

Lucie de Carvalho, John Mullen et Frédéric Armao

I'd sit alone and watch your light My only friend through teenage nights And everything I had to know I heard it on my radio

You made 'em laugh, you made 'em cry You made us feel like we could fly So don't become some background noise

A backdrop for the girls and boys Who just don't know or just don't care And just complain when you're not there You had your time, you had the power

You've yet to have your finest hour Radio

Roger Taylor, 'Radio Gaga', Queen - The Works, 1984.

1 En 2017, la Charte royale de la BBC, qui détermine le statut constitutionnel et les modalités organisationnelles et opérationnelles des activités audiovisuelles de la Corporation, a été remaniée pour la neuvième fois de son histoire. Une fois encore, cette étape majeure pour l'avenir de cette institution a replacé la BBC sous le feux-croisés des attaques et des critiques, dont se dégagent deux principaux chefs d'accusation: d'une part, le manque présumé d'objectivité éditoriale (bias) et de pluralisme politique et d'autre part, son mode de financement par impôt direct sous la forme de redevance (licence fee). Les assauts portés à la neutralité en matière de théorie de la BBC émanent de tous les bords de l'échiquier politique. Alors que la BBC est, par exemple, accusée par 
des commentateurs conservateurs d'une couverture partiale des politiques d'austérité mises en œuvre par la Coalition et le gouvernement Cameron, le journaliste du Guardian, Gavin Lewis attaque sévèrement la couverture de la crise financière de 2008-2009, qui aurait donné trop de crédit aux voix de la City face à celles de la société civile $^{1}$. La BBC aurait également dépeint de façon biaisée l'arrivée de Jeremy Corbyn à la tête du Parti travailliste, le référendum sur l'indépendance de l'Ecosse de 2016 ou enfin, plus récemment, le Brexit. Pour les commentateurs conservateurs, Auntie Beeb aurait cédé aux sirènes du libéralisme et du populisme social (liberal bias) en donnant une part trop belle à la mouvance des « identity politics $»^{2}$.

2 Depuis janvier 2020, c'est son système universel de redevance qui se retrouve une nouvelle fois en ligne de mire des 30000 signataires de la pétition 'Defund the BBC', selon lesquels la redevance devrait être remplacée par un système d'abonnements afin de permettre aux utilisateurs une plus grande liberté de choix. Symbole ici de l'impact de l'arrivée des plateformes de streaming ou de télévision à la demande dans l'écosystème audiovisuel, cette réforme structurelle constituerait une étape cruciale vers la privatisation du secteur de l'audiovisuel britannique.

3 En somme, si la BBC semble aujourd'hui en prise avec une crise existentielle qui fragilise les fondements et les valeurs mêmes de son fonctionnement et de son statut constitutionnel, celle-ci est loin d'être sans précédents. Bien au contraire, ces accusations sont le produit des tensions qui ont affaibli la Corporation de manière cyclique, voire endémique, depuis ses premières heures d'existence au début des années 1920. Néanmoins, force est de constater que les attaques du modèle constitutionnel de la BBC se sont faites de plus en plus virulentes depuis le changement de paradigme néolibéral des années 1970-1980. La BBC était à l'époque sous la houlette du directeur-général John Birt (1992-2000), figure polarisante qui, s'il fut le sauveur de la BBC pour certains, en tentant d'adapter la Corporation aux nouvelles injonctions néolibérales, fut pour d'autres «le Dalek de Downing Street ${ }^{3}$, le fossoyeur de ses valeurs fondamentales de service public.

\section{La BBC : une institution à la trajectoire unique}

4 Il n'en demeure pas moins que la $B B C$ constitue une organisation particulièrement unique et exceptionnelle. De phénomène technoculturel à trésor national ${ }^{4}$, celle qui deviendra séculaire dans deux ans constitue une véritable institution. De par son histoire et sa trajectoire bien particulière, la $\mathrm{BBC}$ fut une pionnière dans le développement technologique et culturel de la communication au XXe siècle. L'historien Asa Briggs n'hésita d'ailleurs pas à apparenter cette épopée politicotechnologique, qui fut souvent romantisée, à une seconde 'révolution Gutenberg'. Pour le théoricien de la littérature britannique, Todd Avery, le 14 novembre 1922 marqua sans nul doute le jour où « le visage de la communication humaine changea de façon irrévocable au Royaume-Uni $»^{5}$.

Dans ce numéro, Renée Dickason se propose de revenir sur la genèse de ce service public de radiodiffusion exceptionnel. Son article souligne l'aventure passionnante que vivaient les techniciens et producteurs qui découvrirent la sensation de parler à des milliers de personnes, chacune chez elle, ainsi que les tâtonnements du gouvernement face à la mise en place d'un cadre légal inédit pour l'audiodiffusion, technologie qui semblait bien prometteuse mais dont peu de gens pouvaient en imaginer l'avenir. 
Institution progressivement tentaculaire, la $\mathrm{BBC}$ a rapidement joui d'une réputation internationale, en devenant un mégaphone d'une amplitude de communication et de dissémination formidable, reflétée par l'origine étymologique du terme même de broadcast, grâce à un réseau qui transcenda rapidement les frontières, notamment à travers l'Empire Service, instauré en 1932. L'étude menée ici par Houcine Msaddek sur la station de radio BBC Arabic à partir de 1938, permettra d'apprécier l'influence de la Corporation sur les cultures locales et régionales mais aussi sa qualité de vecteur du soft power britannique à l'international. L'auteur examine en détail l'image que la BBC voulait projeter du Royaume-Uni et de l'Empire, et surtout la contribution essentielle à la construction d'une chaîne populaire apportée par une série de recrues locales, égyptiennes palestiniennes ou soudanaises. Sa contribution aide à équilibrer les récits de l'histoire du World Service très souvent restés centrés sur les activités londoniennes de la Corporation.

7 Le succès de la $\mathrm{BBC}$ au sein des foyers britanniques fut quasi immédiat, comptant au bout de quatre années de fonctionnement en 1926, près de deux millions d'utilisateurs. La BBC devint un modèle de service de radiodiffusion puis d'audiovisuel de qualité pour ses voisins européens, une source fiable et reconnue de productions culturelles et d'information. Comme les différents articles de ce numéro le démontreront, la BBC va au cours de son histoire s'ériger en une véritable référence culturelle et l'icône d'une certaine forme de britannicité, qu'il conviendra néanmoins de définir au fil des analyses proposées. Comme pour toute aïeule, la BBC aujourd'hui intime une certaine forme de respect et de nostalgie pour un passé plus reluisant et glorieux, où le Royaume-Uni dominait les ondes, et pas uniquement celles des mers. Mais aujourd'hui, Auntie Beeb serait pour certains aussi devenue "un vieux meuble que l'on balade à droite, à gauche ", comme souligne le journaliste Nick Fraser ${ }^{6}$, un objet du patrimoine familial, dont personne n'ose se défaire. Ici, les parallèles entre la BBC et le NHS sont fort nombreux - car derrière les débats autour du futur de la BBC, c'est toute la notion de 'service public' qui se retrouve mise au pilori.

8 En effet, le statut, la place et la fonction de la BBC en tant qu'institution publique ont donné lieu à des débats passionnés et une forte polarisation dans les médias comme dans les sphères politiques ou universitaires, reflet de perspectives idéologiques divergentes autour des limites entre secteur privé et service public, mais surtout de la compréhension même de ce dernier. Souvent tributaires du contexte idéologique de par ses liens intestins et ambigus avec le pouvoir politique central, l'éthique et la fonction de service public de la BBC se sont fondés sur des conventions, des valeurs et des pratiques, souvent tacites, qui ont été, tout au cours de son histoire, redéfinies, questionnées, renégociées, à l'instar de la définition même du concept plus englobant de 'service public de l'audiovisuel'.

9 Malgré une apparente transparence, le concept de 'service public' est fondamentalement le « reflet de son temps $»^{7}$, qui semble concilier «bien commun » (public good) et intérêt national (national interest). Il n'en demeure pas moins " insaisissable ${ }^{8}$, du fait des nombreuses ambiguïtés émanant du statut constitutionnel de la BBC, tel qu'il a été établi dans sa première Charte Royale de 1926. Son statut fut davantage forgé comme le calotype du système de radiodiffusion alors en pleine expansion aux Etats-Unis. La BBC fonctionne depuis comme une institution de nature hybride, étant indépendante des instances politiques (dite " at arm's length ", afin de ne pas être considérée comme une créature politique), tout en restant tributaire du 
gouvernement pour son financement et son existence même - un type d'organisation aujourd'hui plus connu sous le nom de Quango. Par conséquent, le statut de la BBC l'ancre depuis sa naissance dans une zone grise, « un entre-deux entre pouvoir public et pouvoir privé, entre Etat et individus, entre propagande et savoir $»$.

De fait donc, le concept de service public n'a jamais véritablement fait l'objet d'une définition légale ni constitutionnelle clairement formulée; aucun repère constitutionnel ni aucune décision judiciaire n'en a jamais établi les limites de façon normative :

The absence of constitutional principles and the pragmatism and informality of regulation does not mean that concepts of public service broadcasting do not exist in the UK, but rather they are to be found not in general statements but in cultural expectations given expression in the detailed codes and regulatory practices of the authorities. ${ }^{10}$

11 Par conséquent, son bagage symbolique et conceptuel n'a cessé d'évoluer et d'être redéfini par les acteurs eux-mêmes, au gré des rapports des commissions d'enquêtes parlementaires sur les activités nationales de radiodiffusion et audiovisuelles (du rapport Sykes au rapport Peacock de 1986) : dans le présent numéro, Daniel Ruff porte un regard diachronique sur ces sources institutionnelles riches, complexes, et aux corollaires parfois inattendus. Ce concept a également été le produit d'un travail d'autodéfinition de la part des instances dirigeantes de la Corporation même, et notamment des figures de proue de l'institution, les directeurs-généraux, avec en premier lieu, John Reith, dont l'influence fondatrice sera ici explorée en détails par Trevor Harris, qui n'hésite pas à explorer les aspects les plus choquants du personnage, dont son admiration pour le gouvernement de Hitler jusqu'à la veille de la guerre.

12 Selon Reith, la mission première de la $B B C$ en tant que service public était résumée par la trinité dorénavant bien connue de "to inform, educate and entertain». Comme le rappelle Raymond Williams ${ }^{11}$, l'idéal de service public ainsi greffé sur le système de radiodiffusion/audiovisuel pendant ses années de formation (1920-1950), trouve ses racines dans l'acceptation victorienne du terme «service» tel qu'il fut défini par le mouvement progressiste de la dernière partie du XIXe siècle. L'idée était que les institutions publiques se devaient d'être animées par un sens du devoir social et moral (" moral purpose » et « social duty »), un idéal d'ailleurs institutionnalisé dans la fonction publique britannique. Williams précise également que le service public se devait ainsi d'être un instrument progressiste non seulement social mais également culturel, justifiant ainsi la mobilisation de l'autorité de l'Etat non seulement dans les domaines de la santé ou des infrastructures, mais également dans ceux de la culture et de l'éducation, dans le but de servir l'intérêt national défini comme la paix sociale à travers l'atténuation des fractures et des tensions entre les différentes classes sociales.

\section{La BBC et débats historiographiques : de la rareté à l'abondance}

13 Compte tenu de son histoire exceptionnelle et de son rôle central dans la société britannique, la $\mathrm{BBC}$ a fait l'objet d'un travail historiographique particulièrement riche. A l'approche du centenaire de la première diffusion radiophonique, le travail historiographique autour de la $\mathrm{BBC}$ connaît actuellement un regain majeur d'intérêt 
scientifique, s'étoffant de nouvelles ressources, dont le projet A Hundred Voices that Made the $\mathrm{BBC}^{12}$. Ce projet ambitieux et novateur, porté par l'historien britannique David Hendy met en avant les trésors de l'histoire orale de la BBC, qu'il présentera dans l'interview réalisée en ouverture de ce numéro. David Hendy expliquera les défis et les espoirs représentés par l'écriture d'une histoire qui veut trouver un lectorat de masse, tout en évitant autant que possible l'hagiographie.

'une des grandes richesses de l'étude de l'histoire de la BBC est qu'elle se loge à la croisée de différents champs disciplinaires, entre histoire culturelle, histoire des médias et sciences et philosophie politique, par exemple. Comme le soulignera John Mullen, ce kaléidoscope d'histoires permet de soulever un vaste éventail de questionnements. Il cherchera, chez les historiens de l'art ou de la technologie, chez des experts en histoire sociale ou culturelle, des interrogations qui pourront éclairer notre vision de la $\mathrm{BBC}$ et son rôle dans la société britannique, en mettant les émissions dans toute leur variété au centre de ses réflexions.

Il est difficile de ne pas mentionner l'œuvre titanesque de l'historien britannique Asa Briggs qui proposa entre 1961 et 1995 une histoire de la BBC en cinq volumes, de sa naissance aux années $1980^{13}$. Son premier ouvrage répondait alors à une demande spécifique de la BBC qui commandita ainsi sa propre biographie. Briggs obtenu ainsi un accès privilégié à ses archives et documents internes. Il est important de souligner le contexte dans lequel ce vaste chantier fut lancé dans la mesure où le milieu des années 1950 coïncide avec la remise en question de son statut de monopole à travers l'arrivée de la concurrence d'ITV, et l'émergence de nouvelles tensions autour de l'ingérence gouvernementale et du devoir de réserve de l'institution, exacerbées par la crise de Suez.

Force est de constater que Briggs écrivit alors une histoire institutionnelle par excellence, centrée sur les $B B C$ men qui ont forgé les valeurs de l'institution, ainsi que sur les événements politiques et les idées qui ont façonné l'évolution de la Corporation dans une perspective quasi téléologique. Si ce travail scientifique est aujourd'hui encore d'une valeur inestimable, il frise néanmoins le panégyrique de la Corporation.

$17 \mathrm{Au}$ cours des années 1970, les analyses historiques restent encore très centrées sur les instances dirigeantes de la Corporation et ses relations avec l'Etat et/ou les intérêts privés ${ }^{14}$. Néanmoins, elles s'attachent davantage à explorer les tensions et les sources de conflits et dissentions entre ces différents pôles de pouvoir, afin de s'interroger sur l'évolution de la notion de service public dans une perspective qui reste toutefois institutionnelle. Dans ce numéro, Mélanie Dupéré et Elodie Gallet reviennent sur deux situations-clés de l'histoire d'après-guerre, lors desquelles la BBC s'est trouvée une nouvelle fois confrontée aux menaces d'ingérence étatique, d'une part, lors de la crise de Suez de 1956, et d'autre part, lors de l'escalade de la violence en Irlande du Nord des années 1960 aux gouvernements Thatcher.

Les années 1980-1990 marquent toutefois une véritable rupture dans l'approche scientifique de l'histoire de l'audiovisuel britannique. Sous l'influence galvanisante des British Cultural Studies qui donnent à la culture populaire, au quotidien, à l'ordinaire et aux médias leurs lettres de noblesse ${ }^{15}$, l'historiographie de la BBC connaît un véritable renouvellement des approches. Différents chercheurs s'intéressent alors à la manière dont la radio et la télévision ont contribué à questionner et bouleverser les normes sociales et les représentations, et à légitimer de nouvelles pratiques sociales, participant ainsi au développement des Media Studies à travers l'étude des programmes 
et des productions audiovisuelles. Une véritable histoire culturelle, libérale et anti paternaliste de la $\mathrm{BBC}$ prend ainsi son essor à travers les travaux de Paddy Scannell et David Cardiff notamment ${ }^{16}$, qui mettent à l'honneur les programmes de divertissement (soap operas, les comédies, les émissions musicales, le sport, par exemple), au même titre que les émissions plus traditionnelles, comme les documentaires, les œuvres de fiction ou les journaux télévisés.

Dans la lignée de cette approche, Peter Lewis, professor of media and culture à la London Metropolitan University, montrera comment les équipes créatives ont travaillé à faire entendre la voix des travailleurs ordinaires à la radio de la $\mathrm{BBC}$, malgré la tradition de la Corporation établie par Reith qui avait sévèrement restreint l'accès au microphone aux seuls individus jugés compétents pour le faire. Lewis présente notamment les programmes Voice of the People de Richard Kelly (1954) ou Radio Ballads de Ewan MacColl et Peggy Seeger (1958). Ces programmes documentaires incarnent ainsi une volonté d'utiliser les ondes radios comme véhicule de nouvelles formes d'expression populaire, malgré une marge de manœuvre créative souvent étroite au sein d'une BBC encore largement acquise à l'élitisme, avec laquelle ces artistes devront négocier.

Joy Leman analysera la production de fictions radiodiffusées et télévisées dans les années 1950 et 1960 du point de vue de leur traitement des femmes et de la manière dont celles-ci s'adressaient à la classe ouvrière. En outre, Susannah O'Carroll offrira un nouveau regard sur le programme des Wednesday Plays, soulignant elle aussi que si les producteurs de ce programme bénéficiaient d'une certaine autonomie d'action, leur positionnement et leur approche créative étaient parfois source aigue de tensions avec leurs supérieurs au sein de la BBC.

21 En outre, les sitcoms ont été depuis les années 1950 d'une très grande importance aux téléspectateurs britanniques, et cela jusqu'à nos jours. L'omniprésence de l'humour dans la culture britannique est bien connue, et cela aurait été fort dommage que notre numéro n'ait rien à dire sur ce phénomène primordial. Pierre-François Peirano s'intéressera donc aux séries comiques des années 1980, «Yes, Minister » et «Yes, Prime Minister », des fictions qui mettent en scène le personnage de Jim Hacker, un homme politique naïf et perdu, dépassé par ses conseillers plus intelligents et plus cyniques. Il tentera d'évaluer l'ambition de ces séries, entre proposition de réforme des institutions politiques et satire universelle dénuée de véritable dynamique contestataire. Il rappellera également la dimension pédagogique de ces deux séries, qui entendent familiariser les spectateurs avec de nombreux codes et processus à l'œuvre dans les couloirs du pouvoir.

Richard Witts, Reader à l'Université de Edge Hill, complètera ce panorama en livrant une étude de la politique de programmation musicale de la $B B C$ à la lumière de la réorganisation des chaînes de radio de 1967, un moment charnière qui souligna le rôle crucial de la musique dans les débats autour de la spécialisation de la structure de BBC Radio.

23 Ainsi nous présentons des études de différents genres d'émission - fictions, comédies, ou documentaires. A partir de ces différentes études de cas de programmes, ces articles explorent la manière dont producteurs et auteurs se sont adaptés et ont dû composer, avec plus ou moins de succès, avec les impératifs de neutralité éditoriale de la Corporation, afin d'aborder un vaste éventail de questionnements, tout en modernisant les codes des différents formats présentés. 

sociale qui va venir soulever des questionnements nouveaux sur les structures de domination et dynamiques d'inclusivité autour des paramètres de classe, d'ethnicité et de genre ${ }^{17}$. Laura Carter reviendra ainsi le travail de l'historienne et présentatrice Rhoda Power entre 1922 et 1957, afin d'étudier le rôle confié aux femmes dans les politiques éducatives de la $\mathrm{BBC}$, et également d'évaluer la manière dont ce secteur d'activité de la $\mathrm{BBC}$ reflétait les idées dominantes de l'époque concernant la diffusion des connaissances et la stratification sociale. Les émissions éducatives de la BBC à l'intention des établissements scolaires marquèrent des générations d'écoliers et collégiens britanniques ${ }^{18}$. Or, la $\mathrm{BBC}$ entreprit à plusieurs reprises des initiatives ambitieuses en matière des cours pour adultes - d'alphabétisation, de formation à l'informatique et d'autres. Allan Jones, Senior Lecturer à l'Open University reviendra sur cette histoire complexe et tourmentée.

Cette nouvelle histoire sociale s'inscrit dans la lignée de l'approche portée par l'école marxiste et les chercheurs poststructuralistes qui avaient ainsi promu l'importance de «l'histoire d'en bas » et des pratiques matérielles à charge idéologique. Dans la même dynamique, d'autres auteurs, comme Hajkowski ou Harvey et Robins, portent alors un regard plus anthropologique sur les notions d'identité, de britannicité, de nation et de régionalisme ${ }^{19}$. Stéphanie Bory reviendra sur l'impact majeur, quoique peut-être inattendu, de la BBC sur la construction du nationalisme gallois.

26 La fin des années 2000 s'est enfin accompagnée d'un renouveau d'analyses plus théoriques et conceptuelles autour des fonctions symboliques et institutionnelles de la $\mathrm{BBC}$, dans une perspective plus militante de défense du modèle britannique de service public de l'audiovisuel. Revenant sur le changement de paradigme des années 1970-1980, Tom Mills et Jean Seaton portent notamment un regard très critique sur les réformes néolibérales des services publics, qui auraient sérieusement entravé la capacité de la $\mathrm{BBC}$ à protéger son impartialité et son indépendance et à ainsi remplir sa mission démocratique ${ }^{20}$. Dans ce numéro, Simon Dawes proposera également d'étudier la manière dont les dynamiques de concurrence et de libéralisation ont contribué à déconstruire le concept de service public, en lui substituant progressivement celui d'intérêt public.

\section{BBC et démocratie}

Ce numéro se propose donc de contribuer à l'analyse du concept de service public de l'audiovisuel tel qu'il s'est incarné dans la BBC de 1922 à 1995. Il s'agira ici de s'interroger sur la manière dont $\mathrm{la} \mathrm{BBC}$ et ses activités ont contribué à la déconstruction ou recomposition des concepts d'opinion publique, de citoyenneté culturelle, de sphère publique et de démocratie au sens plus large. Il n'est en effet pas anodin que la BBC ait été instituée alors même que se déployait enfin la démocratie de masse au Royaume-Uni à travers le suffrage universel entre 1918 et 1928. Les articles présentés ici permettront donc de définir comment le développement de la démocratie britannique de masse s'est retrouvé intimement lié à son secteur de l'audiovisuel qui de fait - en a également été fonction. Comme le démontreront les articles présentés dans ce numéro, la fonction démocratique du service public de l'audiovisuel soulèvera des questionnements fondamentaux quant à son indépendance théorique face aux influences politiques, aux dangers inhérents à son statut initial de monopole, aux

Revue Française de Civilisation Britannique, XXVI-1 | 2021 
impératifs de neutralité éditoriale et de retenue, et aux évolutions du concept "d'intérêt public ».

Ce numéro portera enfin sur l'évolution du « public » et du caractère « universel » de la mission tripartite de la BBC. Il importera également d'explorer la manière dont la BBC et ses programmes ont tenté de transcender les binarités entre élitisme et populaire, entre programmes de niche et programmes grand public, afin de "parler à tous et toutes ", tout en prenant en considération des groupes sociaux et économiques aux caractéristiques et attentes différentiées. De fait, la visibilité progressive donnée aux différents publics et à leurs attentes va tester la capacité de la BBC à s'adapter à la diversité des publics et à se faire le médiateur de ces identités plurielles. Ici se posera également la question des tensions entre centralisation et représentations/ constructions des identités nationales. Comme le définit Anderson dans son célèbre ouvrage Imagined Communities ${ }^{21}$, les médias en général et les services de l'audiovisuel en particulier constituent des éléments cruciaux dans la construction des identités nationales fantasmées et des sentiments de cohésion et d'appartenance, qui favorisent l'unité d'une communauté donnée ('communal belonging') à travers la ritualisation des productions culturelles nationales radiodiffusées et télévisées. «

En somme, il importera d'insister sur l'importance de décentrer le regard, et de cesser de considérer la BBC comme un ensemble monolithique, dont les choix, les valeurs, les ambitions, et les pratiques n'auraient été dictés que par des considérations managériales ou institutionnelles. Au contraire, une perspective en coupe, plus centrée sur les acteurs et les programmes dans toute leur diversité d'opinion et de positionnement, permettra de donner véritablement chair et matérialité à une institution trop souvent désincarnée, et cela dans toutes ses nuances, ses contradictions et ses ambiguïtés.

\section{NOTES}

1. Gavin Lewis, "The Broken BBC: From Public Service to Corporate Power ", The Monthly Review 67:1 (1 avril 2016).

2. Robin Aitken, Can we Trust the BBC? (Londres, Bloomsbury, 2013) et The Noble Liar and Why the $B B C$ Distorts the News to Promote a Liberal Agenda (Londres, Biteback Publishing, 2018).

3. Rachel Sylvester, "The Rise of John Birt, the Dalek of Downing Street ", The Telegraph (10 janvier 2005).

Le titre fait ici référence aux Daleks, les robots extraterrestres malfaisants de la série de sciencefiction de la BBC, Doctor Who.

4. "One of this nation's most treasured institutions », dira ainsi le député Craig Tracey sur sa page professionnelle en ligne: https://www.craigtracey.co.uk/bbc-charter-review, consultée le 26 juillet 2020.

5. "The character of human communication changed irrevocably in Britain" Todd Avery, Radio Modernism: Literature, Ethics, and the BBC, 1922-1938 (Aldershot, Ashgate Publishing Limited, 2006), p. 1.

6. « an old piece of furniture [which is] kicked around». 
7. " a creature of its time ", Stuart Hall, « Which Public, Whose Service? » dans Wilf Stevenson (dir.), All Our Futures: The Changing Roles and Purposes of the BBC (Londres, British Film Institute, 1993), p. 33.

8. Paddy Scannell, " Public Service Broadcasting ", dans Andrew Goodwin et Garry Whannel (dir.), Understanding Television (Abington, Routledge, 1990), p. 11.

9. «that critical liminal space between public and private powers, state and people, propaganda and knowledge ", Georgina Born, "Reflexivity and Ambivalence: Culture, Creativity and Government in the BBC » Cultural Values 6:2 (2002), pp. 65-90.

10. Tony Prosser, « Public Service Broadcasting and Deregulation in the UK » European Journal of Communication 7:2 (1992), p. 179.

11. Raymond Williams, The Long Revolution (Londres, Chattos \& Windus, 1961).

12. Voir : https://www.bbc.com/historyofthebbc/100-voices

13. Asa Briggs, History of Broadcasting in the United Kingdom: The Birth of Broadcasting 1896-1927, vol 1, (Oxford, Oxford University Press, 1961). --- The Golden Age of Wireless, 1965; --- The War of Words, 1970; --- Sound and Vision, 1979; --- Competition, 1995.

14. Tom Burns, The BBC: Public Institution and Private World (Londres, Macmillan, 1977); Nicholas Garnham, Structures of Television (Londres, British Film Institute, 1978); James Curran et Jean Seaton, Power Without Responsibility: The Press and Broadcasting in Britain (Abington, Routledge, 1985).

15. Stuart Hall, Dorothy Hobson, Andrew Lowe et Paul Willis (dir.), Culture, Media, Language (Londres, Hutchinson, 1980).

Dorothy Hobson, Crossroads: Drama of a Soap Opera (Londres, Methuen, 1982).

Horace Newcomb (dir.), Television: The Critical View (Oxford, Oxford University Press, 1987).

Justin Lewis, « Decoding TV News », dans Phillip Drummond et Richard Paterson (dir.), Television in Transition (Londres, British Film Institute, 1985).

David Morley, Family Television: Cultural Power and Domestic Leisure (Londres, Comedia, 1986).

Michael O'Shaughnessy, « Box Pop: Popular Television and Hegemony », dans Andrew Goodwin et Garry Whannel (dir.), Understanding Television (Abington, Routledge, 1990).

16. Paddy Scannell et David Cardiff, A Social History of British Broadcasting, 1922-1939 (Oxford, Blackwell, 1991).

David Hendy, Life on Air: A History of Radio Four (Oxford, OUP, 2007).

Joe Moran, Armchair Nation: An Intimate History of Britain in front of the TV (Londres, Profile Books Ltd, 2013).

Charlotte Higgins, This New Noise: The Extraordinary Birth and Troubled Life of the BBC (Londres, Guardian Faber Limited, 2015).

17. Gavin Schaffer, The Vision of a Nation - Making Multiculturalism on British Television, 1960-80 (Londres, Palgrave Macmillan, 2014).

Kate Murphy, Behind the Wireless: A History of Early Women at the BBC, (Londres, Palgrave Macmillan, 2016).

Douglas Kerr, « Orwell's BBC Broadcasts: Colonial Discourse and the Rhetoric of Propaganda » Textual Practice 16:3 (2002), pp. 473-490.

18. On peut trouver certaines émissions de $B B C$ Schools en ligne, comme cet exemple racontant l'histoire de la police http://www.youtube.com/watch?v=4koVQxo4Cis

19. Sylvia Harvey et Kevin Robins, « Voices and Places: The BBC and Regional Policy » Political Quarterly 65:1 (1994).

Thomas Hajkowski, The BBC and National Identity in Britain, 1922-53 (Manchester, Manchester University Press. 2010).

20. Steven Barnett et Jean Seaton, « Why the BBC Matters: Memo to the New Parliament about a Unique British Institution » The Political Quarterly 81:3 (2010).

Chris Carter, "Cultures of Strategy: Remaking the BBC, 1968-2003", Business History 55:7 (2003), 
pp. 1228-1246.

Jean Seaton, Pinkoes and Traitors, 1974-1987 (Londres, Profile Books, 2017), 2e édition.

Tom Mills, The BBC: Myth of a Public Service (Londres, Verso, 2020), 2e édition.

21. Benedict Anderson, Imagined Communities: Reflections on the Origins and Spread of Nationalism (Londres, Verso, 1983). 